\title{
Core Schemas in Youth at Clinical High Risk for Psychosis
}

\author{
Jacqueline Stowkowy and Lu Liu \\ University of Calgary, Canada
}

\author{
Kristin S. Cadenhead \\ UCSD, La Jolla, USA \\ Tyrone D. Cannon
}

Yale University, New Haven, USA

Barbara A. Cornblatt

Zucker Hillside Hospital, Long Island NY, USA

Thomas H. McGlashan

Yale University, New Haven, USA

Diana O. Perkins

University of North Carolina, Chapel Hill, USA

\author{
Larry J. Seidman \\ Harvard Medical School at Beth Israel Deaconess Medical Center and Massachusetts General \\ Hospital, Boston, USA \\ Ming T. Tsuang \\ UCSD, La Jolla, USA
}

Reprint requests to Jacqueline Stowkowy, Mathison Centre for Mental Health Research and Education, University of Calgary, 3280 Hospital Drive NW, Calgary, Alberta T2N 4Z6, Canada. E-mail: stowkowy@ucalgary.ca 
Elaine F. Walker

Emory University, Atlanta, USA

Scott W. Woods

Yale University, New Haven, USA

Carrie E. Bearden

UCLA, Los Angeles, USA

Daniel H. Mathalon

UCSF, San Francisco, USA

Robert Heinssen

National Institute of Mental Health, Bethesda, USA

\author{
Jean Addington
}

University of Calgary, Canada

Background: Schema Theory proposes that the development of maladaptive schemas are based on a combination of memories, emotions and cognitions regarding oneself and one's relationship to others. A cognitive model of psychosis suggests that schemas are crucial to the development and persistence of psychosis. Little is known about the impact that schemas may have on those considered to be at clinical high risk (CHR) of developing psychosis. Aims: To investigate schemas over time in a large sample of CHR individuals and healthy controls. Method: Sample included 765 CHR participants and 280 healthy controls. Schemas were assessed at baseline, 6 and 12 months using the Brief Core Schema Scale (BCSS). Baseline schemas were compared to 2-year clinical outcome. Results: CHR participants evidenced stable and more maladaptive schemas over time compared to controls. Schemas at initial contact did not vary amongst the different clinical outcome groups at 2 years although all CHR outcome groups evidenced significantly worse schemas than healthy controls. Although there were no differences on baseline schemas between those who later transitioned to psychosis compared to those who did not, those who transitioned to psychosis had more maladaptive negative self-schemas at the time of transition. Associations between negative schemas were positively correlated with earlier abuse and bullying. Conclusions: These findings demonstrate a need for interventions that aim to improve maladaptive schemas 
among the CHR population. Therapies targeting self-esteem, as well as schema therapy may be important work for future studies.

Keywords: Clinical high risk, psychosis, schemas, beliefs, NAPLS

\section{Introduction}

A cognitive model of psychosis proposes that pre-existing beliefs about the self and others may play a role in the development and persistence of psychosis, and that these effects have been observed over and above depressed or anxious mood (Garety, Bebbington, Fowler, Freeman and Kuipers, 2007). Such pre-existing beliefs about the self are known as schematic beliefs or schemas. Young and colleagues "Schema Theory" states that early maladaptive schemas are created based on a combination of memories, emotions and cognitions regarding oneself and one's relationship to others. They propose that early maladaptive schemas develop in childhood when an environment does not meet the needs of the child due to experiences such as abuse, neglect, hostility or criticism (Young, Klosko and Weishaar, 2003). The emergence of interest in schemas and psychosis originally derived from a non-clinical sample that showed that there were strong associations between negative schemas about self and others and levels of paranoia (Fowler et al., 2006). More recently, it has been demonstrated that, after controlling for depression, patients with schizophrenia evidence significantly more maladaptive schemas compared to healthy controls (Bortolon, Capdevielle, Boulenger, GelyNargeot and Raffard, 2013).

If, indeed, schemas play a role in the development of psychosis, examining schemas in those who are known to be at clinical high risk (CHR) of developing psychosis (Addington and Heinssen, 2011) could offer new insights. However, little is known about the role of schemas in young people at CHR. One study investigated a sample of undergraduate students with elevated schizotypy scores and found that positive schizotypy was associated with negative schemas, and negative schizotypy was associated with diminished positive schemas (Barrantes-Vidal et al., 2013). A second study found that maladaptive schemas appear to be evident in those seeking help for psychosis across various clinical groups (i.e. first episode of psychosis patients, CHR participants and help seeking controls) when compared to individuals who only endorse minimal schizotypal experiences (Taylor et al., 2013). Lastly, in a small study with CHR individuals, it was demonstrated that maladaptive schemas mediated the role between experiencing social defeat and more severe attenuated positive symptoms (Stowkowy and Addington, 2012).

The aims of this study are: first, to examine schemas in a large sample of CHR individuals both longitudinally and in comparison to healthy controls; second, to determine if there is an association between early childhood trauma and schemas; third, to assess, in a sub-sample, whether schemas at initial contact are related to later clinical outcome.

We hypothesize that: (1) CHR participants will evidence significantly more maladaptive schemas compared to controls; (2) schemas will be stable over time; and (3) maladaptive schemas within the CHR group will be related to a history of trauma. Our exploratory hypothesis is that those who go on to develop psychosis may have more maladaptive schemas. 


\section{Method}

Sample

The sample consisted of 765 CHR participants (436 male, 329 female) and 280 healthy controls (141 male, 139 female). All participants were recruited as part of the NIMH funded North American Prodrome Longitudinal Study 2 (NAPLS 2) and were drawn from the participating NAPLS2 sites (Emory University, Harvard University, University of Calgary, University of California at Los Angeles, University of California at San Diego, University of North Carolina, Yale University, and Zucker Hillside Hospital). The NAPLS2 project was established to investigate predictors and mechanisms of conversion to psychosis. All participants were help-seeking individuals referred from various sources, including health care agencies, educators, and self-referrals. Potential participants would undergo a telephone screen and then, if applicable, be invited to attend an in-person evaluation. Further description of ascertainment methods has been well described in detail elsewhere (Addington et al., 2012). All CHR participants met the Criteria of Prodromal Syndromes (COPS) using the Structured Interview for Prodromal Syndromes (SIPS) (McGlashan, Walsh and Woods, 2010). Participants were excluded if they met criteria for any current or lifetime axis I psychotic disorder, IQ less than 70, or past or current history of a clinically significant central nervous system disorder. In addition, control participants were also excluded if they had a first degree relative with a current or past psychotic disorder.

A subsample of individuals (556 CHR, 246 healthy controls) who had completed the final 2-year assessment of the NAPLS project by December 2013 were included in order to examine the association of initial schemas to later clinical outcome. This sub-sample included 71 participants (48 male, 23 female) who had transitioned to psychosis, three of whom transitioned several months after the 2-year follow-up.

\section{Measures}

The Structured Interview for Prodromal Syndromes (SIPS) and the Scale for Assessment of Prodromal Symptoms (SOPS) (McGlashan et al., 2010) were used to determine criteria for a prodromal syndrome and to determine severity of attenuated positive symptoms. The Brief Core Schema Scale (BCSS) (Fowler et al., 2006), a self-report scale, was used to assess negative and positive schemas. The BCSS has 24 items concerning beliefs about the self and others that are assessed on a 5-point rating scale. Four scores are obtained: negative-self, positive-self, negative-others, and positive-others, each with a range of 0 to 24 (a higher score on the negative items indicates more maladaptive schemas, whereas a higher score on the positive items indicates less maladaptive schemas). This scale has been demonstrated to be valid in a CHR population (Addington and Tran, 2009). Additional clinical measures included the Calgary Depression Scale for Schizophrenia (Addington, Addington and Maticka-Tyndale, 1993; Addington, Shah, Liu and Addington, 2014) and an adapted version of the Childhood Trauma and Abuse Scale (Janssen et al., 2004). This scale enquires about trauma and abuse before the age of 16. Respondents answer yes or no to ever experiencing any of the following before age 16: psychological or physical bullying, emotional, physical, psychological or sexual abuse.

Clinical status at the 2-year follow-up was determined in the following way: 1) healthy control (does not meet criteria for prodromal risk syndrome); 2) remission (scores of 2 or less 
on the five positive symptoms on the SOPS scale); 3) symptomatic (not currently meeting criteria for a prodromal risk syndrome but having ratings of 3-5 on any one of the five positive symptoms on the SOPS); 4) prodromal progression (currently meeting criteria for one of the at risk syndromes; APSS; the emergence or worsening of non-psychotic level disturbances in thought content, thought processes or perceptual abnormalities over the past year, GRD; either a first degree relative with a psychotic disorder or the subject having schizotypal personality disorder (SPD) plus at least a 30\% drop in functioning on the General Assessment of Functioning (GAF) scale in the past 12 months, BIPS; the emergence of at least one psychotic symptom that is too brief to meet criteria for a psychotic disorder and 5) psychotic (currently meeting criteria for a psychotic disorder or evidencing scores of 6 on one or more positive symptoms of the SOPS).

\section{Procedures}

The study was approved by Institutional Review Boards at all eight sites participating in NAPLS. Participants provided informed consent or assent (parental informed consent for minors). Participants were assigned a clinical rater who conducted semi-structured interviews. Raters were experienced research clinicians who demonstrated adequate reliability at routine reliability checks. Gold standard post-training agreement on determining the prodromal diagnoses was excellent (kappa $=0.90$ ). Follow up assessments were conducted at 6 and 12 months after baseline. If an individual transitioned to psychosis, an additional assessment was completed.

\section{Statistical analyses}

Baseline demographics were compared between CHR and control groups using the Student $t$-test for continuous variables and chi-square tests for categorical variables. To account for any missing data and intra-participant correlation over time, mixed effects models for longitudinal data were used to compare the difference for the BCSS total scores (negative-self, negativeothers, positive-self, positive-others) between CHR and control groups at baseline, 6-month follow-up, and 12-month follow-up. Spearman's correlation coefficients were calculated to determine the correlation between schemas and the clinical variables of positive symptoms, negative symptoms, depression, and trauma. The five groups based on 2-year clinical status (1 -healthy control; 2 - remission; 3 - symptomatic; 4 - prodromal, and 5 - psychotic) were compared on baseline schemas using a one-way ANOVA. The $t$-test for two independent samples was used to determine the differences in baseline schemas between those who transitioned to psychosis and those who did not transition to psychosis by 2 years. Lastly, the $t$-test for two independent samples was also used to determine the differences between the schemas of those at the time of transition to psychosis and the schemas at the 2-year assessment of those who did not transition.

\section{Results}

Demographic characteristics are summarized in Table 1. Control participants were slightly older and had significantly more years of education than CHR participants. CHR females reported significantly more negative schemas about the self and negative schemas about others 
Table 1. Demographic characteristics

\begin{tabular}{|c|c|c|c|c|}
\hline Variable & $\begin{array}{l}\text { Controls } \\
n=280\end{array}$ & $\begin{array}{l}\text { CHR } \\
n=765\end{array}$ & $\begin{array}{l}\text { Test } \\
\text { statistic }\end{array}$ & $\begin{array}{l}\text { Effect } \\
\text { size }\end{array}$ \\
\hline & \multicolumn{2}{|c|}{ Mean $(S D)$} & $t$ & $d$ \\
\hline Age in years & $19.65(4.67)$ & $18.47(4.24)$ & $3.68^{*}$ & 0.26 \\
\hline \multirow[t]{2}{*}{ Years of education } & $12.68(3.58)$ & $11.30(2.82)$ & $5.84^{*}$ & 0.43 \\
\hline & \multicolumn{2}{|c|}{ Number $(\%)$} & $X^{2}$ & Cramer's V \\
\hline \multicolumn{5}{|l|}{ Sex: } \\
\hline Male & $141(50.4 \%)$ & $436(57.0 \%)$ & 3.65 & 0.06 \\
\hline Female & $139(49.6 \%)$ & $329(43.0 \%)$ & & \\
\hline \multicolumn{5}{|l|}{ Race: } \\
\hline First Nations & $4(1.4 \%)$ & $13(1.7 \%)$ & 4.96 & 0.07 \\
\hline Asian & $30(10.7 \%)$ & $55(7.2 \%)$ & & \\
\hline Black & $49(17.5 \%)$ & $118(15.4 \%)$ & & \\
\hline Latin America/Middle East/White & $167(59.6 \%)$ & $478(62.6 \%)$ & & \\
\hline Native Hawaiian or Pacific Islander & $1(0.4 \%)$ & $3(0.4 \%)$ & & \\
\hline Interracial & $29(10.4 \%)$ & $97(12.7 \%)$ & & \\
\hline \multicolumn{5}{|l|}{ Marital status: } \\
\hline Single never married & $266(95.0 \%)$ & $721(94.9 \%)$ & 0.01 & 0.003 \\
\hline Other & $14(5.0 \%)$ & $39(5.1 \%)$ & & \\
\hline \multicolumn{5}{|l|}{ Currently working } \\
\hline Yes & $129(46.1 \%)$ & $190(25.1 \%)$ & $42.38^{*}$ & 0.20 \\
\hline No & $151(53.9 \%)$ & $568(74.9 \%)$ & & \\
\hline \multicolumn{5}{|l|}{ Currently enrolled as a student } \\
\hline Yes & $227(81.1 \%)$ & $626(82.5 \%)$ & 0.28 & 0.02 \\
\hline No & $53(18.9 \%)$ & $133(17.5 \%)$ & & \\
\hline
\end{tabular}

${ }^{*} p<0.01$

than CHR males (mean $=7.24, S D=5.85$ vs. mean $=5.74, S D=5.23, t=-3.80, p<.0001$, and mean $=8.67, S D=6.37$ vs. mean $=7.38, S D=5.94, t=-3.05, p<.01$, respectively). There were no differences in negative schemas between males and females in the healthy control group. Males and females did not differ in positive schemas for either controls or CHR participants.

Our longitudinal analysis in the mixed effects model was based on $N=714 \mathrm{CHR}$ and $N$ $=273$ healthy controls. There were no differences in baseline schemas between those who were lost to follow-up and those who remained. Since the groups differed significantly on depression as rated by CDSS (baseline; $t=-26.56, p<.0001 ; 6$ months; $t=-17.32, p<.0001$; 12 months; $t=-14.36, p<.0001$ ) we controlled for depression at each time point in the mixed effects model. There were no within group differences in schemas overtime for either the control or CHR groups. As displayed in Table 2 the groups differed on both negative and positive schemas at each time point.

Results of the Spearman's correlations in the CHR group are presented in Table 3. As in previous studies, depression was highly correlated with schemas and was controlled for in the correlations between schemas and trauma. Only 676 of the CHR participants had trauma data. A Bonferroni correction was also applied and thus only those correlations with a $p$ value greater than 0.0001 were considered to be significant. Negative-self schemas were 
Table 2. Comparisons between control and prodromal group at baseline, 6 months and 12 months

\begin{tabular}{|c|c|c|c|c|c|c|c|c|c|c|c|c|}
\hline \multirow[b]{2}{*}{ BCSS } & \multicolumn{4}{|c|}{ Baseline } & \multicolumn{4}{|c|}{ 6-months } & \multicolumn{4}{|c|}{ 12- months } \\
\hline & $\mathrm{M}^{*}(\mathrm{SE})$ & $t$ & $p$ & 95\% C.I. & $\mathrm{M}^{*}(\mathrm{SE})$ & $t$ & $p$ & 95\% C.I. & $\mathrm{M}^{*}(\mathrm{SE})$ & $t$ & $p$ & $95 \%$ C.I. \\
\hline Negative self & $\begin{array}{r}-3.5 \\
(0.43)\end{array}$ & -8.19 & $<.0001$ & $\begin{array}{l}-4.72 \\
-2.28\end{array}$ & $\begin{array}{r}-2.83 \\
(0.46)\end{array}$ & -6.1 & $<.0001$ & $\begin{array}{l}-4.16 \\
-1.51\end{array}$ & $\begin{array}{r}-2.90 \\
(0.47)\end{array}$ & -6.18 & $<.0001$ & $\begin{array}{l}-4.24 \\
-1.56\end{array}$ \\
\hline Negative others & $\begin{array}{r}-3.18 \\
(0.62)\end{array}$ & -5.14 & $<.0001$ & $\begin{array}{l}-4.95 \\
-1.41\end{array}$ & $\begin{array}{r}-2.10 \\
(0.67)\end{array}$ & -3.13 & .02 & $\begin{array}{l}-4.02 \\
-0.18\end{array}$ & $\begin{array}{r}-2.21 \\
(0.68)\end{array}$ & -3.25 & .02 & $\begin{array}{l}-4.15 \\
-0.27\end{array}$ \\
\hline Positive self & $\begin{array}{c}3.61 \\
(0.62)\end{array}$ & 5.85 & $<.0001$ & $\begin{array}{l}1.85 \\
5.37\end{array}$ & $\begin{array}{c}4.77 \\
(0.67)\end{array}$ & 7.13 & $<.0001$ & $\begin{array}{l}2.86 \\
6.68\end{array}$ & $\begin{array}{c}3.59 \\
(0.68)\end{array}$ & 5.3 & $<.0001$ & $\begin{array}{l}1.66 \\
5.23\end{array}$ \\
\hline Positive others & $\begin{array}{c}2.72 \\
(0.61)\end{array}$ & 4.48 & $<.0001$ & $\begin{array}{l}0.99 \\
4.46\end{array}$ & $\begin{array}{c}2.90 \\
(0.66)\end{array}$ & 4.39 & $<.0001$ & $\begin{array}{l}1.01 \\
4.79\end{array}$ & $\begin{array}{c}2.77 \\
(0.67)\end{array}$ & 4.15 & $<.0001$ & $\begin{array}{l}0.86 \\
4.68\end{array}$ \\
\hline
\end{tabular}

Notes: BCSS = Brief Core Schema Scale; C.I. = confidence interval;

$\mathrm{M}^{*}=$ represents the least squares means estimated by the generalized linear mixed models 
Table 3. Correlations between schemas, symptoms and trauma

\begin{tabular}{|c|c|c|c|c|}
\hline Measure & $\begin{array}{l}\text { Negative } \\
\text { self } \\
N=676\end{array}$ & $\begin{array}{l}\text { Negative } \\
\text { others } \\
N=676\end{array}$ & $\begin{array}{l}\text { Positive } \\
\text { self } \\
N=676\end{array}$ & $\begin{array}{l}\text { Positive } \\
\text { others } \\
N=676\end{array}$ \\
\hline \multicolumn{5}{|c|}{ Pearson $r$} \\
\hline$\overline{\mathrm{CDSS}}$ & $0.60^{*}$ & 0.29* & $-0.42^{*}$ & $-0.21^{*}$ \\
\hline \multicolumn{5}{|c|}{ After controlling for depression (baseline CDSS scores) } \\
\hline SOPS -P & 0.12 & $\mathrm{0.23}^{*}$ & 0.07 & -0.06 \\
\hline SOPS -N & 0.04 & 0.03 & -0.11 & -0.07 \\
\hline Physical bullying & 0.08 & 0.12 & -0.02 & -0.09 \\
\hline Psych. bullying & 0.11 & $0.17^{*}$ & -0.11 & -0.07 \\
\hline Emotional neglect & 0.10 & 0.07 & -0.01 & -0.09 \\
\hline Psych. abuse & $0.16^{*}$ & 0.08 & -0.03 & -0.07 \\
\hline Physical abuse & $0.13^{*}$ & 0.08 & -0.03 & -0.07 \\
\hline Sexual abuse & 0.05 & 0.09 & -0.02 & -0.12 \\
\hline
\end{tabular}

Notes: ${ }^{*} p<.001[$ EPS] after applying the Bonferroni correction;

CDSS $=$ Calgary Depression Scale for Schizophrenia; SOPS-P $=$ SOPS Total Positive Symptoms, SOPS-N $=$ SOPS Total Negative Symptoms, Psych. $=$ Psychological

Table 4. Current clinical status of groups at 2-year assessment and baseline schemas

\begin{tabular}{|c|c|c|c|c|c|c|}
\hline \multirow[b]{2}{*}{$\begin{array}{l}\text { Two-year } \\
\text { assessment }\end{array}$} & \multicolumn{6}{|c|}{ Baseline schemas } \\
\hline & $\begin{array}{l}\text { Healthy } \\
\text { controls } \\
(N=135)\end{array}$ & $\begin{array}{l}\text { Remission } \\
(N=86)\end{array}$ & $\begin{array}{l}\text { Symptomatic } \\
(N=77)\end{array}$ & $\begin{array}{l}\text { Prodromal } \\
\text { progression } \\
(N=57)\end{array}$ & $\begin{array}{l}\text { Psychotic } \\
(N=66)\end{array}$ & $\begin{array}{l}F \\
\text { Value }\end{array}$ \\
\hline & & Mean $(S D)$ & & & & \\
\hline Negative self & $0.61(1.49)^{\mathrm{a}}$ & $5.83(5.78)$ & $6.10(5.15)$ & $7.86(5.95)$ & $6.86(5.85)$ & $37.69^{*}$ \\
\hline Negative others & $2.65(3.80)^{\mathrm{a}}$ & $7.26(6.06)$ & $9.03(6.12)$ & $7.92(6.02)$ & $7.95(5.43)$ & $24.40^{*}$ \\
\hline Positive self & $17.89(4.45)^{\mathrm{a}}$ & $11.38(5.72)$ & $11.36(5.76)$ & $7.93(6.02)$ & $10.92(6.59)$ & $36.99^{*}$ \\
\hline Positive others & $14.41(4.34)^{\mathrm{a}}$ & $10.19(5.79)$ & $9.52(5.26)$ & $8.86(4.88)$ & $10.12(5.46)$ & $20.14^{*}$ \\
\hline
\end{tabular}

Notes: ${ }^{*} p<.0001 ; \mathrm{a}=$ significantly different from all other groups

positively correlated with psychological abuse and physical abuse. Negative-other schemas were positively correlated with psychological bullying and total positive symptoms. There were no correlations between trauma and positive schemas.

The subsample of individuals that had completed a 2-year assessment and also had data on baseline schemas consisted of $N=66$ individuals who transitioned to psychosis, $N=220$ who did not and $N=135$ healthy controls. An examination of the differences in baseline schemas among the different clinical outcome groups at 24 months revealed that all of the CHR groups differed from controls but did not differ from one another (see Table 4). There were no differences in baseline schemas between those who transitioned to psychosis and those who did not transition for both negative and positive schemas.

Fifty-five of those who made the transition to psychosis had completed the schema questionnaire at the time of transition to psychosis. The schemas of those who transitioned to psychosis $(N=55)$ were compared to the 2 -year schemas of those who did not $(N=204)$. 
Those who transitioned to psychosis reported significantly more negative-self schemas (mean $=5.78, S D=5.89$ ) compared to the schemas at the two-year assessment of those who did not transition $($ mean $=3.80, S D=4.61 ; t=-2.31, p<0.05)$.

\section{Discussion}

As hypothesized, across all time points CHR individuals evidenced more maladaptive schemas compared to controls. This remained after controlling for levels of depression, which provides further support that these schemas are operating over and above depressed mood (Garety et al., 2007). Not only did the CHR individuals endorse more maladaptive schemas such as "I am unloved", "I am worthless" but they also reported fewer positive schemas (i.e. "I am talented", "I am valuable", "I am good") relative to the healthy controls. This is consistent with results of the UK study (Taylor et al., 2013). Additionally, as hypothesized, schemas remained stable over time for both CHR individuals and controls.

Symptoms were not typically correlated to schemas with the one exception being a correlation between more severe attenuated psychotic symptoms and more maladaptive schemas about others. With respect to early trauma, as hypothesized, CHR individuals who reported experiencing increased childhood psychological and physical abuse endorsed more negative schemas about the self and those CHR individuals who reported experiencing childhood psychological bullying endorsed more negative beliefs about others. This might suggest that childhood bullying is contributing to the development of negative schemas about others (i.e. "other people are bad", "other people are hostile", "other people are unforgiving"). On the other hand, these might also manifest as a result of more severe attenuated positive symptoms. For example, if one feels more suspicious of others they might also believe that they are "unworthy". This research is correlational and as such is limited in terms of understanding directionality of this finding, but as our measure of trauma is reported before the age of 16, it may be reasonable to suggest the trauma may have occurred first, at least for some participants. This offers support for the Schema Theory, as well as a further emphasis on prevention. Intervening earlier and attempting to address issues such as bullying continues to be an important area that should be considered.

At the 2-year outcome, healthy controls evidenced less negative schemas and more positive schemas compared to every other clinical group (i.e. remission, symptomatic, prodromal and psychotic). Interestingly, this suggests that for those at CHR maladaptive schemas may be operating independently, despite improvement in or complete remission of attenuated symptoms. While there were no differences in baseline schemas between those who later transitioned to psychosis and those who did not, there were differences between schemas at the time of transition to psychosis and schemas at the 2-year assessment of those who did not transition. Those who transitioned evidenced more negative-self schemas (i.e. "I am unloved", or "I am worthless"). This suggests that although schemas appeared to be stable over time in this CHR group and typically more maladaptive than healthy controls, in fact those individuals who transition to psychosis, at least during the time of transition to psychosis, began to feel worse about themselves. Therefore, the instability of these core schemas during the transition to psychosis might be an important area to review in future research.

There are limitations to our study. It is possible that although we did control for depression there are other variables contributing to our findings for which we did not control. Our measure of trauma did not provide details on the onset, frequency or duration of trauma. 
The strengths of our study are that, unlike previous research, we had a large sample of CHR individuals and healthy controls. We also investigated schemas over time and included findings on both negative and positive schemas. This is also the first study to our knowledge that has looked at schemas in a CHR population and their relation to transition to psychosis.

In conclusion, this study found that CHR individuals evidence significantly more maladaptive schemas compared to controls, and that these are relatively consistent over time. There appears to be a worsening of these schemas for individuals at the time of transition to psychosis. Taken together, these findings may provide further evidence that therapies targeting self-esteem may be an important intervention for this population. Additionally, Schema Therapy, developed by Young and colleagues (Young et al., 2003), is a broad integrative model that incorporates aspects of cognitive behavioural therapy (CBT) and other psychodynamic models, and aims to improve coping styles that may have manifested due to these maladaptive schemas. It has four main concepts (i.e. early maladaptive schemas, coping styles, schema domains and schema modes) with early maladaptive schemas being central to the model. ST has shown promising findings when used throughout various psychopathologies (Masley, Gillanders, Simpson and Taylor, 2012) and may also provide a possible intervention. Determining the best or most appropriate treatment is a question for future research; nonetheless, it is clear that maladaptive schemas play a role in psychosis and should be considered in both clinical and research settings.

\section{Acknowledgements}

This study was supported by the National Institute of Mental Health (grant U01MH081984 to Dr Addington; grants U01 MH081928; P50 MH080272; Commonwealth of Massachusetts SCDMH82101008006 to Dr Seidman; grants R01 MH60720, U01MH082022 and K24 MH76191 to Dr Cadenhead; grant U01MH081902 to Dr Cannon; P50 MH066286 (Prodromal Core) to Dr Bearden; grant U01MH082004to Dr Perkins; grant U01MH081988 to Dr Walker; grant U01MH082022 to Dr Woods; and UO1 MH081857-05 grant to Dr Cornblatt. The NIMH had no further role in study design; in the collection, analysis and interpretation of data; in the writing of the report; and in the decision to submit the paper for publication.

Thanks are due to the following members of the NAPLS group: T. Raedler, L. McGregor, D. Marulanda, L. Legere, C. Marshall, E. Denny, E. Fitton, K. Smith, J.A. Addington (University of Calgary); T. Alderman, K. Shafer, I. Domingues, A. Hurria, H. Mirzakhanian (UCSD); B. Walsh, J. Saksa, N. Santamauro, A. Carlson, J. Kenney, B. Roman (Yale University); K. Woodberry, A.J. Giuliano, W. Stone, J.M. Rodenhiser, L. Tucker, R. Serur, G. Min, R. SzentImrey (Beth Israel Deaconess Medical Center/Harvard); C. Bearden, P. Bachman, J. Zinberg, S. DeSilva, A. Andaya, S. Uguryan (UCLA); J. Brasfield, H. Trotman, (Emory University); A. Pelletier, K. Lansing, H. Mates, J. Nieri, B. Landaas, K. Graham, E. Rothman, J. Hurta, Y. Sierra (University of North Carolina); A. Auther, R. Carrion, M. McLaughlin, R. Olsen (Zucker Hillside Hospital).

\section{References}

Addington, D., Addington, J. and Maticka-Tyndale, E. (1993). Assessing depression in schizophrenia: the Calgary Depression Scale. British Journal of Psychiatry, 163, 39-44. 
Addington, J., Cadenhead, K. S., Cornblatt, B. A., Mathalon, D. H., McGlashan, T. H., Perkins, D. O., et al. (2012). North American Prodrome Longitudinal Study (NAPLS 2): overview and recruitment. Schizophrenia Research, 142, 77-82.

Addington, J. and Heinssen, R. (2011). Prediction and prevention of psychosis in youth at clinical high risk. Annual Review of Clinical Psychology, 8, 269-289.

Addington, J., Shah, H., Liu, L. and Addington, D. (2014). Reliability and validity of the Calgary Depression Scale for Schizophrenia (CDSS) in youth at clinical high risk for psychosis. Schizophrenia Research, 153 (1-3), 64-67.

Addington, J. and Tran, L. (2009). Using the brief core schema scales with individuals at clinical high risk of psychosis. Behavioural and Cognitive Psychotherapy, 37, 227-231.

Barrantes-Vidal, N., Gross, G. M., Sheinbaum, T., Mitjavila, M., Ballespi, S. and Kwapil, T. R. (2013). Positive and negative schizotypy are associated with prodromal and schizophrenia-spectrum symptoms. Schizophrenia Research, 145, 50-55.

Bortolon, C., Capdevielle, D., Boulenger, J. P., Gely-Nargeot, M. C. and Raffard, S. (2013). Early maladaptive schemas predict positive symptomatology in schizophrenia: a cross-sectional study. Psychiatry Research, 209, 361-366.

Fowler, D., Freeman, D., Smith, B., Kuipers, E., Bebbington, P., Bashforth, H., et al. (2006). The Brief Core Schema Scales (BCSS): psychometric properties and associations with paranoia and grandiosity in non-clinical and psychosis samples. Psychological Medicine, 36, 739-749.

Garety, P. A., Bebbington, P., Fowler, D., Freeman, D., and Kuipers, E. (2007). Implications for neurobiological research of cognitive models of psychosis: a theoretical paper. Psychological Medicine, 37, 1377-1391.

Janssen, I., Krabbendam, L., Bak, M., Hanssen, M., Vollebergh, W., de Graaf, R., et al. (2004). Childhood abuse as a risk factor for psychotic experiences. Acta Psychiatrica Scandinavia, 109, 38-45.

Masley, S. A., Gillanders, D. T., Simpson, S. G. and Taylor, M. A. (2012). A systematic review of the evidence base for Schema Therapy. Cognitive Behavior Therapy, 41, 185-202.

McGlashan, T., Walsh, B. C. and Woods, S. W. (2010). The Psychosis Risk Syndrome: handbook for diagnosis and follow-up. New York: Oxford University Press.

Stowkowy, J. and Addington, J. (2012). Maladaptive schemas as a mediator between social defeat and positive symptoms in young people at clinical high risk for psychosis. Early Intervention Psychiatry, 6, 87-90.

Taylor, H. E., Stewart, S. L., Dunn, G., Parker, S., Fowler, D. and Morrison, A. P. (2013). Core schemas across the continuum of psychosis: a comparison of clinical and non-clinical groups. Behavioural and Cognitive Psychotherapy, 42, 718-730.

Young, J. E., Klosko, J. S. and Weishaar, M. E. (2003). Schema Therapy: a practitioner's guide. New York: Guilford Press. 